\title{
Baseline cognitive functions among elderly patients with localised breast cancer ${ }^{\text {许, 访访 }}$
}

\begin{abstract}
Marie Lange ${ }^{\mathrm{a}, \mathrm{b}, \mathrm{c}, \mathrm{d}, \mathrm{e}, \mathrm{f}, 1}$, Bénédicte Giffard ${ }^{\mathrm{a}, \mathrm{b}, \mathrm{c}, \mathrm{d}, 2}$, Sabine Noal ${ }^{\mathrm{f}, \mathrm{k}, 3}$, Olivier Rigal ${ }^{\mathrm{g}, \mathrm{h}, 4}$, Jean-Emmanuel Kurtz ${ }^{\mathrm{i}, 5}$, Natacha Heutte ${ }^{\mathrm{e}, \mathrm{f}, \mathrm{j}, 1}$, Christelle Lévy ${ }^{\mathrm{k}, 6}$, Djelila Allouache ${ }^{\mathrm{k}, 6}$, Chantal Rieux ${ }^{\mathrm{f}, 3}$, Johan Le Fel ${ }^{\mathrm{g}, 4}$, Aurélie Daireaux ${ }^{\mathrm{f}, 3}$, Bénédicte Clarisse ${ }^{\mathrm{f}, 3}$, Corinne Veyret ${ }^{\mathrm{h}, 4}$, Philippe Barthélémy ${ }^{\mathrm{i}, 5}$, Nadine Longato ${ }^{\mathrm{i}, 5}$, Francis Eustache ${ }^{\mathrm{a}, \mathrm{b}, \mathrm{c}, \mathrm{d}, 2}$, Florence Joly ${ }^{b, e, f, 1, *, *}$
\end{abstract}

a INSERM, U1077, Caen, France

${ }^{\mathrm{b}}$ Normandie Université, UMR-S1077, Caen, France

${ }^{\mathrm{c}}$ Ecole Pratique des Hautes Etudes, UMR-S1077, Caen, France

${ }^{\mathrm{d}} \mathrm{CHU}$ de Caen, U1077, Caen, France

e INSERM, U1086, Caen, France

${ }^{\mathrm{f}}$ Unité de Recherche Clinique, Centre François Baclesse, Caen, France

${ }^{\mathrm{g}}$ Service des soins de support, Centre Henri-Becquerel, Rouen, France

${ }^{\mathrm{h}}$ Département d'Oncologie médicale, Centre Henri-Becquerel, Rouen, France

i Département d'hématologie et d'oncologie, Hôpitaux universitaires de Strasbourg, Strasbourg, France

${ }^{\mathrm{j}}$ UFR des sciences pharmaceutiques, Université de Caen Basse-Normandie, Caen, France

${ }^{\mathrm{k}}$ Comité Sein, Centre François Baclesse, Caen, France

${ }^{1} \mathrm{CHU}$ de Caen, Service d'Oncologie, Caen, France

\footnotetext{
Data in this paper were presented in part as an oral communication at the ASCO Annual Meeting (Chicago, 31st May 2013-4th June 2013), as a poster at the meeting of the Federation of the European Societies of Neuropsychology (Berlin, 12-14th September 2013), and as a poster at the conference of International Cognition and Cancer Taskforce (Seattle, 10-12th February 2014).

站败 The registration identification number of this clinical trial is NCT01333735.

* Corresponding author at: Service de recherche clinique, Centre François Baclesse, BP 5026, 3 Av. Général Harris, 14076 Caen Cedex 05, France. Tel.: +33 231455002; fax: +33 231455097.

E-mail addresses: m.lange@baclesse.fr (M. Lange), benedicte.giffard@unicaen.fr (B. Giffard), s.noal@baclesse.fr (S. Noal), olivier.rigal@chb. unicancer.fr (O. Rigal), J-Emmanuel.KURTZ@chru-strasbourg.fr (J.-E. Kurtz),n.heutte@baclesse.fr (N. Heutte), c.levy@baclesse.fr (C. Lévy), d.allouache@baclesse.fr (D. Allouache), c.rieux@baclesse.fr (C. Rieux), johan.lefel@chb.unicancer.fr (J.L. Fel), aurelie.daireaux@neuf.fr (A. Daireaux), b.clarisse@baclesse.fr (B. Clarisse), corinne.veyret@chb.unicancer.fr (C. Veyret), philippe.barthelemy@chru-strasbourg.fr (P. Barthélémy), nadine.longato@chru-strasbourg.fr (N. Longato), neuropsycho@chu-caen.fr (F. Eustache), f.joly@baclesse.fr (F. Joly).

${ }^{1}$ Address: U1086 INSERM-UCBN, Centre François Baclesse, BP 5026, 3 Av. Général Harris, 14076 Caen Cedex 05, France. Tel.: +33 231455002; fax: +33231455097.

${ }_{2}^{2}$ Address: Inserm-EPHE-UCBN U1077, CHU Côte de Nacre - CS 30001, F-14033 Caen Cedex, France. Tel.: +33 231065197; fax: +33 231065198 .

${ }^{3}$ Address: Service de recherche clinique, Centre François Baclesse, BP 5026, 3 Av. Général Harris, 14076 Caen Cedex 05, France. Tel.: +33 231455002; fax: +33231455097.

${ }^{4}$ Address: Service des soins de support, Centre Henri Becquerel, Rue d'Amiens, 76038 Rouen Cedex 1, France. Tel.: +33 232082918; fax: +33 232082936.

${ }^{5}$ Address: Hôpitaux Universitaires de Strasbourg, Departement d'hematologie et d'oncologie, Hôpital de Hautepierre, 1, Av Molière, BP 49, 67200 Strasbourg, France. Tel.: +33 388128314 .

${ }^{6}$ Address: Comité Sein, Centre François Baclesse, BP 5026, 3 Av. Général Harris, 14076 Caen Cedex 05, France. Tel.: +33 231455050; fax: +33 231455097.
} 


\section{KEYWORDS \\ Cognition disorders \\ Breast neoplasms \\ Ageing \\ Neuropsychology \\ Quality of life}

\begin{abstract}
Purpose: Cognitive deficits (CD) are reported among cancer patients receiving chemotherapy, but may also be observed before treatment. Though elderly patients are expected to be more prone to present age-related $\mathrm{CD}$, poor information is available regarding the impact of cancer and chemotherapy on this population. This study assessed baseline cognitive functions (before adjuvant treatment) in elderly early stage breast cancer (EBC) patients.

Methods: Women $>65$ years-old with newly diagnosed EBC were included in this prospective study. Episodic memory, working memory, executive functions and information processing speed were assessed by neuropsychological tests. Questionnaires were used to assess subjective $\mathrm{CD}$, anxiety, depression, fatigue, quality of life and geriatric profile. Objective CD were defined using International Cognition and Cancer Task Force criteria. A group of elderly women without cancer coupled with published data related to healthy women were used for comparison (respectively to subjective and objective CD).

Results: Among the 123 elderly EBC patients ( $70 \pm 4$ years) included, $41 \%$ presented objective $\mathrm{CD}$, which is greater than expected in healthy population norms (binomial test $P<.0001$ ). Verbal episodic memory was mainly impaired ( $21 \%$ of patients). No correlation was observed between objective CD and cancer stage or geriatric assessment. Subjective CD only correlated with verbal episodic memory $(P=.01)$.

Conclusions: This is the first large series assessing baseline cognitive functions in elderly EBC patients. More than $40 \%$ presented objective CD before any adjuvant therapy, which is higher than what is reported among younger patients. Our results reinforce the hypothesis that age is a risk factor for CD in EBC patients.

(c) 2014 Elsevier Ltd. All rights reserved.
\end{abstract}

\section{Introduction}

Beyond difficulties with memory, attention and concentration reported by cancer patients, it has become increasingly apparent that cytotoxic drugs given for non-central nervous system tumours might induce cognitive side-effects. This phenomenon - called 'chemobrain' - has been particularly studied among young women treated with chemotherapy for breast cancer. According to the literature, these cognitive troubles could affect $15-50 \%$ of chemotherapy-treated patients and are usually moderate in severity [1]. Nevertheless, recent longitudinal studies revealed that about $20-30 \%$ of breast cancer patients have cognitive impairment before starting adjuvant treatment [2]. This indicates that beside exposure to cytotoxic drugs, other factors including postoperative dysfunctions, psychological distress related with the diagnosis, fatigue, genetic factors and also the biological adverse effects of cancer itself are involved, suggesting an impact of cancer as a whole on cognitive functions $[3,4]$.

The mean patients' age in the previous studies addressing the impact of cancer on cognitive function was less than 65 years. Yet, because cancer increasingly appears among seniors, the impact of ageing on cognitive impairment is a relevant issue. Ageing by itself is known to be associated with some cognitive modifications, comorbidities and functional decline, which may all have an impact on the patients' independence. While both ageing and cancer are expected to have an impact on cognition, biologic processes underlying cancer led to the hypothesis that age-associated declines among cancer patients would not just be parallel to but higher than those of older adults with no cancer history, and that treatment-induced accelerated ageing would be observed only in vulnerable or frail populations [2]. Although a pretreatment cognitive evaluation is a prerequisite to define the part of cognitive chemotherapy-induced impairment, only one study, to our knowledge, addressed this issue especially among elderly breast cancer patients [5].

The aim of the present prospective study was to precisely assess cognitive functioning (objective performances and subjective complaints) among elderly EBC patients before starting adjuvant therapy, and to seek for correlations with mood, fatigue, quality of life and clinical variables.

\section{Patients and methods}

\subsection{Participants}

Newly diagnosed and consecutive elderly women with EBC were recruited from three French Cancer 
comprehensive Centers (Caen, Rouen and Strasbourg) from January 2009 to August 2012.

Inclusion criteria were EBC and age over 65. Exclusion criteria included prior exposure to chemotherapy or radiotherapy, neurological comorbidities, known psychiatric comorbidities which might affect capacity to participate, major cognitive disorders and documented alcohol or drug abuse. Participants with a Mini-Mental State Examination (MMSE) score less than 25 out of 30 suggesting potential pathological ageing were not eligible [6-8] as well as those reporting a formal education less than 5 years (end of the primary school) due to the lack of normative data for such individuals.

Patients were assessed after surgery, but before any adjuvant treatment initiation. They were evaluated with standardised neuropsychological tests, by a graduate neuropsychologist, and through self-report questionnaires. Cognitive performances were compared to published normative data, adjusted for age and/or education. All patients gave their written informed consent to the longitudinal study which was approved by the local ethics committee.

\subsection{Assessment}

The neuropsychological battery included standardised neuropsychological tests assessing four cognitive domains: episodic memory (verbal and visual modalities), working memory, information processing speed and executive functions (Table 1) [7,9-13]. The subjective assessment consisted of a self-report measure of cognitive complaints (Functional Assessment of Cancer Therapy Cognitive Scale - FACT-Cog, version $3[14,15]$ - four subscales: Perceived Cognitive Impairments, Impact on Quality of Life, Comments from Others, and Perceived Cognitive Abilities), assessment of depression (Beck Depression Inventory - BDI [16]), anxiety (Spielberger
State-Trait Anxiety Inventory - STAI [17]), fatigue (Functional Assessment of Chronic Illness TherapyFatigue - FACIT-Fatigue, version 4 [18]) and quality of life (FACT-Breast - FACT-B, version 4 [19] incorporates FACT-General - FACT-G, version 4 [20]). Geriatric assessment included the Geriatric Depression Scale (GDS) [21] (4-item short form), the Instrumental Activities of Daily Living (IADL) [22], the Activities of Daily Living (ADL) [23], the Charlson comorbidity index [24], the number of medications and main previous medical history. Clinical variables were Performance Status (PS), medications with potential impact on cognition (Level 3 on the WHO analgesic ladder, anxiolytics, antidepressant treatments and hypnotics), cancer stage, time since surgery, type of surgery, HER 2 positive and hormone receptor status.

\subsection{Procedure}

Patients completed neuropsychological tests, geriatric scales and some self-report questionnaires (the BDI and the STAI) in a $2 \mathrm{~h}$-session with a neuropsychologist. The other self-report questionnaires were completed by the patients at home.

\subsection{Assessment criteria}

According to the recommendations of the International Cognition and Cancer Task Force [25] and as described previously [26], an index for each patient's baseline overall cognitive function was operationally defined as impaired or not impaired using a 2-part criterion: if patients performed at a $z$-score of $\leqslant 1.5$ standard deviation (SD) on two or more tests, or if they performed at a $z$-score of $\leqslant 2.0$ SDs on a single test, they were classified as impaired. This 2-step approach was designed to minimise the number of potential falsepositive errors resulting from multiple tests and to

Table 1

Neuropsychological tests grouped by main cognitive domains.

\begin{tabular}{|c|c|c|c|}
\hline Cognitive domain & Test & Outcome measure & Range \\
\hline \multicolumn{4}{|l|}{ Episodic memory } \\
\hline Verbal episodic memory & Grober and Buschke procedure [20] & 4 free recalls & $(4 \times) 0-16$ \\
\hline Visual episodic memory & Rey Complex Figure [19] & Recall score & $0-36$ \\
\hline \multirow[t]{4}{*}{ Working memory } & WAIS-III [21]: Arithmetic & Number of resolved problems & $0-22$ \\
\hline & WAIS-III: Digit-span & Correct trials, forward & $0-16$ \\
\hline & & Correct trials, backward & $0-14$ \\
\hline & WAIS-III: Letter-number sequencing & Total correct trials & $0-21$ \\
\hline $\begin{array}{l}\text { Information processing } \\
\text { speed }\end{array}$ & TMT A $[18]$ & Time to complete and errors & $\geqslant 0$ \\
\hline \multicolumn{4}{|l|}{ Executive function } \\
\hline Flexibility & TMT B [18] & $\begin{array}{l}\text { Time to complete and number of perseverative } \\
\text { errors }\end{array}$ & $\geqslant 0$ \\
\hline Information generation & $\begin{array}{l}\text { Verbal fluency [17]: Category (animal) and } \\
\text { Letter P }\end{array}$ & Total score over 2 min & $\geqslant 0$ \\
\hline
\end{tabular}

WAIS, Wechsler Adult Intelligence Scale; TMT, Trail Making test. 
determine the frequency of impairment rather than low performance. By using curves based on the binomial probability distribution [27], we determined that in a battery of eight independent tests approximately 17\% of the population would perform two SDs below the mean on a single test, making $17 \%$ considered as the significant threshold. Like for overall cognitive function, using the classification criteria described above, a cognitive domain was considered as impaired if it included one impaired score.

As a reference of self-report cognitive complaints in the general population, a group of 71 healthy women matched on age and education to the EBC patients included (recruited through local advertisements and among associations) completed the FACT-Cog. Clinically significant symptoms of mood disorders and fatigue were operationally defined as ratings on the BDI $\geqslant 8$ [16], STAI $\geqslant 56$ [17] and FACIT-Fatigue $<37$ [28]. Geriatric profile was established using GDS (0-4, high score $=$ more depression), IADL $(0-8$, low scor$\mathrm{e}=$ no functional status problem) and ADL (0-6, high score $=$ no functional status problem) scores; patients were considered as having a frailty profile if they had at least one alteration of these scores (GDS $>0$, $\mathrm{IADL}>0, \mathrm{ADL}<6$ ).

\subsection{Statistical analysis}

Published normative data, adjusted for age and/or education, were used to convert patients' raw neuropsychological test scores into standardised scores ( $z$ scores; mean, $0 ; \mathrm{SD}, 1)$. Descriptive statistics were generated for the socio-demographic and clinical variables. Comparisons were made by chi square, Student's, and Wilcoxon's tests. The correlations between cognitive complaints and objective cognitive scores and other self-report measures were assessed with Spearman's rank correlation coefficient. Given the large number of correlations performed, a $P$-value $<0.01$ was considered in order to minimise type I error. All analyses were conducted using SAS version 9.3.

\section{Results}

\subsection{Sample characteristics}

Of 221 elderly patients with EBC screened, 11 were ineligible, and 82 were not enrolled in the trial for the following reasons: lack of interest $(n=17)$, too much burden $(n=9)$, travel limitations $(n=17)$, duration of the assessment $(n=10)$, or other reason $(n=29)$. This yielded a $61 \%$ participation rate. Moreover, five patients were excluded from analysis because of a score above the threshold of dementia [8]. Hence, the final sample consisted of 123 patients, whose major characteristics are presented in Table 2 . The majority of elderly patients

Table 2

Demographic and clinical characteristics of all patients $(n=123)$.

\section{Demographic}

Age (years) (mean, SD, range)

Education level (low/middle/high) $(\%)$ (mean, SD)

$70(4.10)[65$ $83]$

$66 / 15 / 18$

$11(2.77)$

Clinical

PS $(\mathrm{WHO}=0)(\%)$

Co-morbidities (\%) Charlson index (0/1-2)

$>3$ co-medications

Medications with potential impact on cognition" $(\%)$

Cancer stage I-II (\%)

Time since surgery (days) (median, range)

Lumpectomy/mastectomy (\%)

Lymph node dissection (\%)

HER 2 positive ( $\%)$

Hormone receptors positive $(\%)$

$\overline{\mathrm{SD} \text {, standard deviation; PS, performance status; WHO, World Health }}$ Organisation.

* Level 3 on the WHO analgesic ladder, anxiolytics, antidepressant treatments and hypnotics.

did not exhibit geriatric comorbidities, was healthy (PS $0=91 \%$ ) and two thirds had a low level of education.

\subsection{Neuropsychological outcomes}

Using the classification criteria described above, $41 \%$ of patients $(51 / 123$, binomial test $P<.0001)$ had impaired overall cognitive function which is significantly more frequent than what would be expected in the general population. Twenty-nine percent (36/123) exhibited impairment on 1 test, whereas $12 \%(15 / 123)$ exhibited impairment on two or more tests. Main impairment was related to visual episodic memory and executive functions ( 21 and $16 \%$ of patients, respectively - cf. Fig. 1). Raw neuropsychological test scores, $z$-scores or standard scores are shown in Table 3.

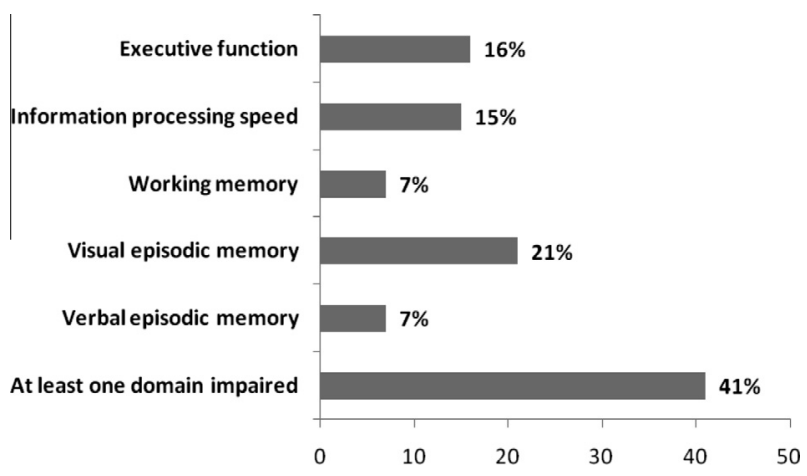

Fig. 1. Distribution of patients according to impaired cognitive domain. The percent of patients with cognitive impairment in each cognitive domain assessed and for at least one impaired cognitive domain. 
Table 3

Neuropsychological test and quality of life outcomes.

\begin{tabular}{|c|c|c|c|c|}
\hline Cognitive scores & No. & Mean & SD & $z$ scores or standard scores \\
\hline \multicolumn{5}{|l|}{ Episodic memory } \\
\hline FR1 & 123 & 8.84 & 1.94 & $0.17(0.88)$ \\
\hline FR2 & 123 & 10.61 & 1.94 & $0.28(0.83)$ \\
\hline FR3 & 123 & 11.66 & 1.87 & $0.21(0.83)$ \\
\hline DFR & 122 & 11.95 & 2.30 & $0.17(1.14)$ \\
\hline Rey recall & 123 & 15.24 & 6.24 & $-1.38(1.27)$ \\
\hline \multicolumn{5}{|l|}{ Working memory } \\
\hline Arithmetic & 122 & 10.11 & 4.38 & $8.80(2.55)$ \\
\hline Digit-span forward & 123 & 7.99 & 2.17 & \\
\hline Digit-span backward & 123 & 4.95 & 1.60 & \\
\hline Digit-span std score & & & & $8.81(3.01)$ \\
\hline Letter-number sequencing & 123 & 7.62 & 2.44 & $8.75(2.54)$ \\
\hline \multicolumn{5}{|l|}{ Information processing speed } \\
\hline TMT A time & 123 & 45.71 & 17.50 & $-0.32(0.78)$ \\
\hline TMT A errors & 123 & 0.17 & 0.46 & $0.27(1.42)$ \\
\hline \multicolumn{5}{|l|}{ Executive function } \\
\hline Semantic fluency score & 123 & 27.75 & 7.03 & $0.10(0.88)$ \\
\hline Phonemic fluency score & 123 & 19.24 & 6.50 & $0.03(1.03)$ \\
\hline TMT B time & 122 & 111.43 & 45.26 & $-0.40(0.67)$ \\
\hline TMT B perseverative errors & 122 & 0.52 & 0.85 & $0.42(1.18)$ \\
\hline \multicolumn{5}{|l|}{ Quality of life scores } \\
\hline FACIT-Fatigue & 111 & 40.02 & 9.16 & \\
\hline FACT-B & 112 & 23.40 & 6.27 & \\
\hline FACT-G global score & 110 & 81.83 & 10.56 & \\
\hline PWB & 110 & 24.54 & 3.31 & \\
\hline SWB & 111 & 19.97 & 4.15 & \\
\hline EWB & 112 & 19.19 & 3.62 & \\
\hline FWB & 112 & 17.96 & 4.35 & \\
\hline \multicolumn{5}{|l|}{$F A C T-C o g$} \\
\hline PCI & 112 & 60.16 & 9.91 & \\
\hline QoL & 106 & 11.63 & 4.06 & \\
\hline Oth & 110 & 15.55 & 1.11 & \\
\hline PCA & 108 & 19.38 & 4.97 & \\
\hline \multicolumn{5}{|l|}{ Anxiety \& depression scores } \\
\hline BDI & 123 & 3.39 & 3.28 & \\
\hline STAI State & 123 & 37.58 & 10.68 & \\
\hline
\end{tabular}

No., number; FR, free recall; DFR, delayed free recall; TMT, trail making test; PWB, physical well-being; SWB, social/family well-being; EWB, emotional well-being; FWB, functional well-being; PCI, perceived cognitive impairment; QoL, impact on quality of life; Oth, comments from others; PCA, perceived cognitive abilities; BDI, beck depression inventory; STAI, spielberger state-trait anxiety inventory.

\subsection{Cognitive complaints}

Healthy subjects had significantly more complaints on Perceived Cognitive Impairments and Perceived Cognitive Abilities FACT-Cog subscales than patients (Fig. 2). However, patients had more complaints than healthy subjects on the subscale Impact on Quality of Life of cognitive impairment $(P<.025)$.

\subsection{Anxiety, depression, fatigue, geriatric and quality of life scores}

Anxiety, depression and severe fatigue were observed in $6 \%, 10 \%$ and $29 \%$ of the patients, respectively. Regarding geriatric scores, $89 \%$ and $87 \%$ of the patients had normal GDS and functional status scores. Quality of life scores are shown in Table 3.

\subsection{Relation between cognitive complaints and neuropsychological scores, anxiety, depression and fatigue}

As shown in Table 4, cognitive complaints were correlated with verbal episodic memory impairment (Perceived Cognitive Impairments subscale, $P<.01$ ) but overall cognitive scores were not correlated with cognitive complaints (the four subscales of the FACT-Cog).

However, three of the four subscales of the FACT-Cog (Perceived Cognitive Impairments, Perceived Cognitive Abilities and Impact on Quality of Life) were significantly correlated with the measures of depression, 

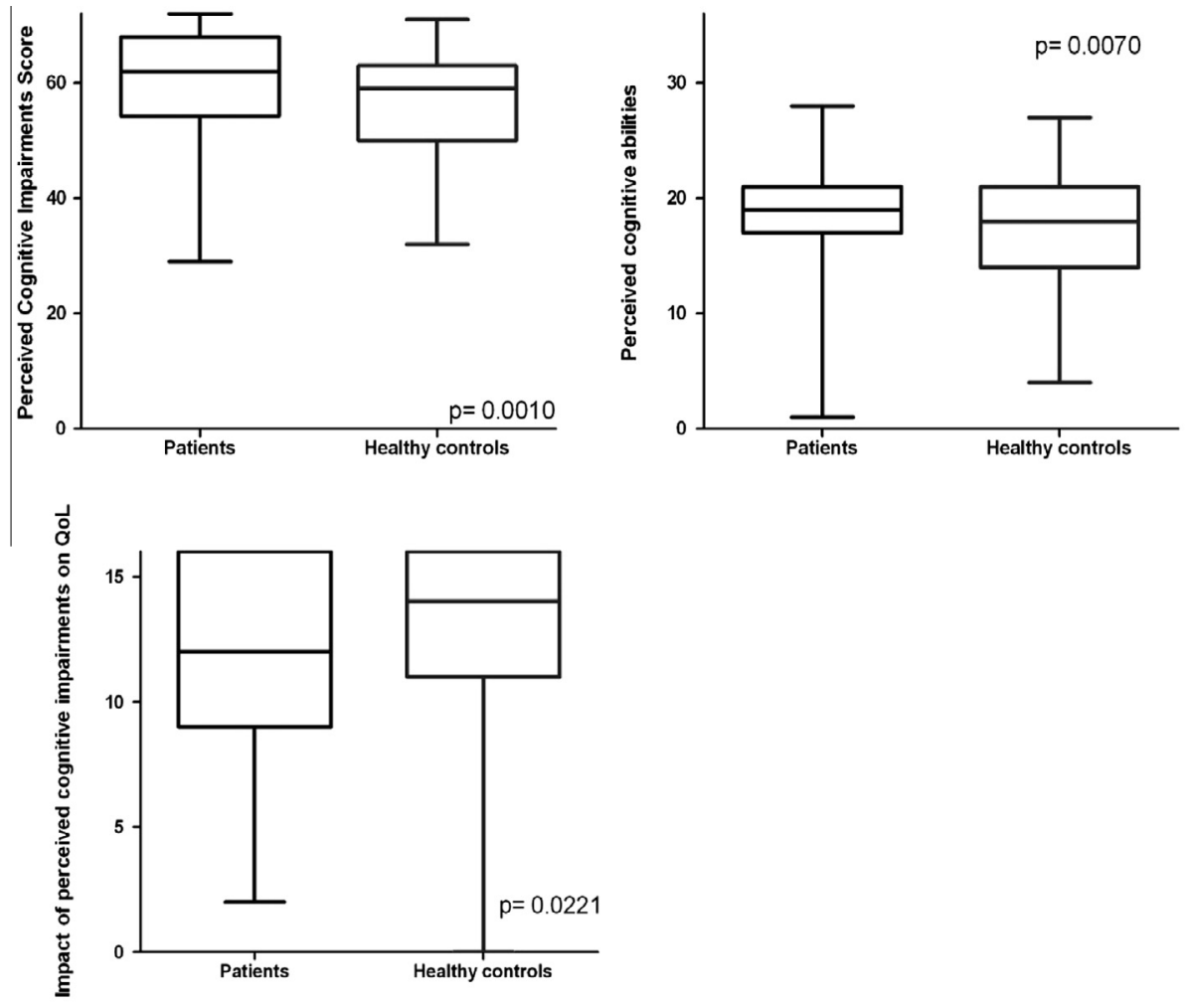

QoL: Quality of Life

Fig. 2. Median Functional Assessment of Cancer Therapy Cognitive Scale (FACT-Cog) scores of patients and healthy subjects. Whiskers' boxplot indicates minimum and maximum and high scores indicate low complaints. There were significant differences between patients and healthy subjects on 2 FACT-Cog subscales. The subscale Comments from Others was not represented because there was no difference between groups.

anxiety and fatigue $(P<.001$; Table 4$)$. Furthermore, the same three subscales were overall significantly associated to quality of life scores (FACT-B and FACT-G; Table 4).

\subsection{Neuropsychological outcomes and clinical characteristics}

Cognitive impairment was not significantly correlated with geriatric profile $(P=0.83)$. Furthermore, there was no correlation between cognitive impairment and Charlson index, PS, medications with potential impact on cognition, cancer stage, time since surgery, type of surgery, hormonal receptor and Her2 status.

\section{Discussion}

This study is the first large series assessing cognitive functions in elderly EBC patients prior to adjuvant treatment. The main result is that, compared to normative data based on age and education, $41 \%$ of the patients had cognitive impairment mainly epitomised by impaired visual episodic memory before any adjuvant treatment which is significantly higher than what would be expected considering healthy population norms. To avoid confusing bias from pre-treatment status to that of chemotherapy-induced impairment, some authors proposed the term 'chemobrain' to be replaced by 'cancerbrain' [30,31]. In this way, evaluation of cognitive functions before treatment appears essential to understand the impact of treatments on cognitive functions of cancer patients especially among elderly patients.

In our study, the proportion of patients exhibiting pre-treatment impairments was higher than the one reported in studies focusing on younger breast cancer patients [45-55 years-old] ranging from $20 \%$ to $30 \%$ [2]. This important finding supports the hypothesis that elderly patients may be more sensitive to the impact of cancer on cognition, and would be consistent with the link between biological processes underlying cancer, ageing, neurodegeneration and a cognitive decline as proposed by Ahles [32]. However, longitudinal studies remain necessary to investigate whether or not cancer therapies accelerate cognitive ageing [32].

In a previous pilot longitudinal study exploring baseline cognitive functioning of elderly patients with breast cancer $(n=28), 11 \%$ of the patients were found to have cognitive impairment before beginning chemotherapy [5]. However, $86 \%$ of the patients had an education level superior to high school (against only $18 \%$ in our 
Table 4

Relations between cognitive complaints and neuropsychological, anxiety, depression, fatigue and quality of life scores.

\begin{tabular}{|c|c|c|c|c|}
\hline \multirow[t]{2}{*}{ Spearman correlation } & \multicolumn{4}{|c|}{ Cognitive complaints } \\
\hline & PCI & PCA & Oth & QoL \\
\hline \multicolumn{5}{|l|}{ Neuropsychological scores } \\
\hline At least 1 domain impaired ( $t$ test $)$ & -0.48 & 0.93 & 0.65 & 0.67 \\
\hline Verbal episodic memory impairment & $-0.31^{*}$ & -0.10 & -0.24 & -0.20 \\
\hline FR1 & 0.23 & 0.23 & 0.16 & 0.24 \\
\hline FR2 & 0.23 & 0.18 & 0.16 & 0.17 \\
\hline FR3 & 0.10 & 0.10 & 0.003 & -0.02 \\
\hline DFR & 0.19 & 0.04 & 0.17 & 0.24 \\
\hline Visual episodic memory impairment (Rey recall) & -0.05 & 0.009 & -0.03 & -0.20 \\
\hline Working memory impairment & $<0.001$ & -0.07 & -0.01 & -0.05 \\
\hline Arithmetic & -0.03 & 0.12 & 0.07 & 0.12 \\
\hline Digit-span & 0.09 & 0.12 & 0.10 & 0.10 \\
\hline Letter-number sequencing & 0.06 & 0.09 & 0.06 & 0.17 \\
\hline Information processing speed impairment & 0.04 & -0.13 & -0.11 & 0.20 \\
\hline TMT A time & -0.06 & -0.10 & 0.05 & 0.01 \\
\hline TMT A errors & -0.04 & -0.08 & -0.10 & 0.21 \\
\hline Executive function impairment & -0.03 & -0.16 & 0.02 & -0.12 \\
\hline Semantic fluency score & 0.05 & 0.18 & -0.09 & 0.03 \\
\hline Phonemic fluency score & -0.06 & 0.06 & -0.10 & 0.20 \\
\hline TMT B time & -0.06 & -0.18 & 0.03 & -0.09 \\
\hline TMT B perseverative errors & -0.18 & -0.08 & -0.17 & -0.12 \\
\hline \multicolumn{5}{|l|}{ Demographic scores } \\
\hline Age (years) & -0.06 & 0.02 & -0.02 & -0.15 \\
\hline Education (years) & 0.05 & 0.13 & 0.23 & 0.20 \\
\hline \multicolumn{5}{|l|}{ Anxiety, depression scores } \\
\hline BDI - depression & $-0.38^{* *}$ & $-0.32^{* *}$ & -0.20 & $-0.32^{* *}$ \\
\hline STAI State - anxiety & $-0.32^{* *}$ & $-0.35^{* *}$ & -0.19 & $-0.34^{* *}$ \\
\hline \multicolumn{5}{|l|}{ Quality of life scores } \\
\hline FACIT-Fatigue & $0.47^{* *}$ & $0.44^{* *}$ & 0.23 & $0.50^{* *}$ \\
\hline FACT-B & $0.26^{*}$ & $0.25^{*}$ & 0.19 & $0.47^{* *}$ \\
\hline FACT-G & $0.33^{* *}$ & $0.47^{* *}$ & 0.22 & $0.40^{* *}$ \\
\hline PWB & 0.25 & $0.34^{* *}$ & 0.15 & $0.39^{* *}$ \\
\hline SWB & 0.13 & $0.27^{*}$ & 0.19 & -0.02 \\
\hline EWB & $0.32^{* *}$ & $0.36^{* *}$ & 0.16 & $0.44^{* *}$ \\
\hline FWB & $0.31^{* *}$ & $0.39^{* *}$ & 0.13 & $0.42^{* *}$ \\
\hline
\end{tabular}

FR, free recall; DFR, delayed free recall; FACT-G subscales, PWB, physical well-being; SWB, social/family well-being; EWB, emotional wellbeing; FWB, functional well-being; PCI, perceived cognitive impairment; PCA, perceived cognitive abilities; QoL, impact on quality of life; Oth, comments from others.

${ }^{*} P<0.01$.

${ }^{* *} P<0.001$.

population), which may have biased the results and underestimated the impact on cognition. Thus, according to the concept of cognitive reserve suggesting that some subjects may cope better than others with brain damage, high education level could reduce the sensitivity of patients to the impact of cancer on cognition [2].

To our knowledge, the present study is unprecedented in exploring differences between elderly breast cancer patients and healthy controls with the FACTCog. For two of the four FACT-Cog subscales, breast cancer patients reported significantly less cognitive complaints (Perceived Cognitive Impairments and Perceived Cognitive Abilities) than healthy controls, but seemed to have more complaints on Impact on Quality of life subscale. One hypothesis could be that patients with breast cancer are more likely to put cognitive impairment into perspective due to the context of the disease, even though those minor difficulties may indeed have an impact on their quality of life significantly.

The present data also suggest that, in accordance with previous studies, cognitive complaint scores were correlated with anxiety, depression and fatigue scores. However, no correlation was found with overall objective cognitive scores [33], except between verbal episodic memory impairment and the Perceived Cognitive Impairments subscale. The latter could allow assessing this cognitive domain, especially regarding memory verbal information retrieval (accounting for one third of this subscale items). These results are consistent with those reported by Ganz and colleagues based on cognitive complaint questionnaire assessing four subscales specific to one cognitive domain, which suggested that subjective cognitive complaints partly reflect objective performance in domain-specific cognitive test [34]. 
In the present study, all patients underwent a selected geriatric assessment. Interestingly, the results showed that the presence of at least one impaired cognitive domain was not significantly correlated with geriatric fragility assessed from GDS and functional status (ADL and IADL). However, the definition of geriatric fragility could be considered as relatively strict (at least one score of the three scales altered), and only a few number of patients were considered as having a geriatric frail profile, which could represent a selection bias of our sample. Furthermore, the large majority of the patients included in our study were in relatively good general health as indicated by the proportion with PS $0(91 \%)$ or Charlson index $0(78 \%)$, suggesting that the proportion of patients with cognitive impairments $(40 \%)$ could have been underestimated. Indeed, nobody can exclude that the proportion of cancer patients with cognitive impairment prior to adjuvant treatment could be higher in a global and more heterogeneous population regarding geriatric conditions.

While the present quality of life-related data appeared to be in the same range as those reported in the overall cancer population [29], cognitive functioning in elderly patients remains an important issue to be taken into account in the decision making of adjuvant treatment.

The lack of direct comparison with a group of healthy subjects remains the main limitation of this study. Furthermore, the population of patients was clinically heterogeneous regarding some characteristics (cancer stage, hormonal receptor status, type of surgery...). Another possible selection bias could be the low number of geriatric scores in our population or the impact of patients' motivation.

In conclusion, this study is the first large series assessing baseline cognitive functions in elderly EBC patients. The main finding is that cognitive impairment prior to adjuvant therapy was more frequent than what is observed or reported in both comparatively healthy elderly subjects and younger breast cancer patients; this reinforces the hypothesis that age-associated decline among cancer patients is pronounced (i.e. age is a risk factor for $\mathrm{CD}$ in breast cancer patients). Evaluation of cognition before treatment is essential to take into account the impact of treatments on cognitive functions, especially among elderly cancer patients. Furthermore, cancer treatments could accelerate the ageing process in a vulnerable or frail population. In this respect, additional research including such baseline assessment is needed to understand, anticipate and manage the shortand long-term effects of cancer therapy on the cognitive function of elderly patients.

\section{Disclosures}

Financial disclosures: Sanofi - France.

\section{Conflict of interest statement}

None declared.

\section{Acknowledgements}

This work was supported by a National grant (Programme Hospitalier de Recherche Clinique, PHRC) and Sanofi - France. We thank all patients for their participation, clinicians of the breast comity, the Cancéropôle Nord-Ouest and the Centre de Traitement des Données du Cancéropôle Nord-Ouest. We also acknowledge the assistance of Hervé Bismut in manuscript preparation.

\section{References}

[1] Joly F, Rigal O, Noal S, Giffard B. Cognitive dysfunction and cancer: which consequences in terms of disease management? Psychooncology 2011;20:1251-8.

[2] Ahles TA. Brain vulnerability to chemotherapy toxicities. Psychooncology 2012;21:1141-8.

[3] Scherling CS, Smith A. Opening up the window into "Chemobrain": a neuroimaging review. Sensors (Basel) 2013;13:3169-203.

[4] Ahles TA, Saykin AJ. Candidate mechanisms for chemotherapyinduced cognitive changes. Nat Rev Cancer 2007;7:192-201.

[5] Hurria A, Rosen C, Hudis C, et al. Cognitive function of older patients receiving adjuvant chemotherapy for breast cancer: a pilot prospective longitudinal study. J Am Geriatr Soc 2006;54:925-31.

[6] Crum RM, Anthony JC, Bassett SS, Folstein MF. Populationbased norms for the mini-mental state examination by age and educational level. JAMA 1993;269:2386-91.

[7] Kalafat M, Hugonot-Diener L, Poitrenaudl J. French standardization of the "Mini Mental State" (MMS) Greco version. Revue de Neuropsychologie 2003;13(2):209-36.

[8] Folstein MF, Folstein SE, Mchugh PR. "Mini-Mental State". A practical method for grading the cognitive state of patients for the clinician. J Psychiatr Res 1975;12:189-98.

[9] Cardebat D, Doyon B, Puel M, et al. Formal and semantic lexical evocation in normal subjects. performance and dynamics of production as a function of sex, age and educational level. Acta Neurol Belg 1990;90:207-17.

[10] Rey A. Manual of copy and memory reproduction test of complex geometric figures. Paris: Éditions Centre De Psychologie Appliquée; 1959.

[11] Reitan R. Validity of trail making tests as an indicator of organic brain damage. Percept Motor Skills 1958;8:271-6.

[12] Van Der Linden M, Adam S, Agniel A, Antérion-Thomas C, Baisset-Mouly C, Coyette F, et al. Assessment of memory impairment. Marseille: Solal; 2004.

[13] Wechsler D. Wechsler adult intelligence scale-III. San Antonio: The Psychological Corporation; 1997.

[14] Joly F, Lange M, Rigal O, et al. French version of the functional assessment of cancer therapy-cognitive function (FACT-Cog) version 3. Supp Care Cancer 2012;20:3297-305.

[15] Wagner LI, Sweet J, Butt Z, et al. Measuring patient self-reported cognitive function: development of the functional assessment of cancer therapy-cognitive function instrument. J Support Oncol 2009; 7:W32-9.

[16] Beck AT, Beamesderfer A. Assessment of depression: the depression inventory. Mod Probl Pharmacopsychiatry 1974;7:151-69.

[17] Spielberger SD. Manual for the state-trait anxiety inventory (STAI). Palo Alto, CA: Consulting Psychologists Press; 1983. 
[18] Yellen SB, Cella DF, Webster K, et al. Measuring fatigue and other anemia-related symptoms with the functional assessment of cancer therapy (FACT) measurement system. J Pain Symptom Manage 1997;13:63-74.

[19] Brady MJ, Cella DF, Mo F, et al. Reliability and validity of the functional assessment of cancer therapy-breast quality-of-life instrument. J Clin Oncol 1997;15:974-86.

[20] Cella DF, Tulsky DS, Gray G, et al. The functional assessment of cancer therapy scale: development and validation of the general measure. J Clin Oncol 1993;11:570-9.

[21] Clement JP, Nassif RF, Leger JM, Marchan F. Development and contribution to the validation of a brief French version of the Yesavage geriatric depression scale. Encephale 1997;23:91-9.

[22] Lawton MP, Brody EM. Assessment of older people: selfmaintaining and instrumental activities of daily living. Gerontologist 1969;9:179-86.

[23] Katz S, Downs TD, Cash HR, Grotz RC. Progress in development of the index of ADL. Gerontologist 1970;10:20-30.

[24] Charlson ME, Pompei P, Ales KL, Mackenzie CR. A new method of classifying prognostic comorbidity in longitudinal studies: development and validation. J Chronic Dis 1987;40: 373-83.

[25] Wefel JS, Vardy J, Ahles T, Schagen SB. International cognition and cancer task force recommendations to harmonise studies of cognitive function in patients with cancer. Lancet Oncol 2011;12: $703-8$.
[26] Wefel JS, Vidrine DJ, Veramonti TL, et al. Cognitive impairment in men with testicular cancer prior to adjuvant therapy. Cancer 2011;117:190-6.

[27] Ingraham LG, Aiken CB. An empirical approach to determining criteria for abnormality in test batteries with multiple measures. Neuropsychology 1996;10:120-4.

[28] Wratten C, Kilmurray J, Nash S, et al. Fatigue during breast radiotherapy and its relationship to biological factors. Int $\mathrm{J}$ Radiat Oncol Biol Phys 2004;59:160-7.

[29] Brucker PS, Yost K, Cashy J, et al. General population and cancer patient norms for the functional assessment of cancer therapy-general (Fact-G). Eval Health Prof 2005;28:192-211.

[30] Hede K. Chemobrain is real but may need new name. J Natl Cancer Inst 2008;100, pp. 162-163, 169.

[31] Hurria A, Somlo G, Ahles T. Renaming "Chemobrain". Cancer Invest 2007;25:373-7.

[32] Ahles TA, Root JC, Ryan EL. Cancer- and cancer treatmentassociated cognitive change: an update on the state of the science. J Clin Oncol 2012;30:3675-86.

[33] Pullens MJ, De Vries J, Roukema JA. Subjective cognitive dysfunction in breast cancer patients: a systematic review. Psychooncology 2010;19:1127-38.

[34] Ganz PA, Kwan L, Castellon SA, et al. Cognitive complaints after breast cancer treatments: examining the relationship with neuropsychological test performance. J Natl Cancer Inst 2013;105:791-801. 